\title{
How Lasing Localized Structures Evolve out of Passive Mode Locking
}

\author{
M. Marconi, ${ }^{1}$ J. Javaloyes, ${ }^{1,2^{*}}$ S. Balle, ${ }^{3}$ and M. Giudici ${ }^{1}$ \\ ${ }^{1}$ Institut Non-Linéaire de Nice, Université de Nice Sophia Antipolis, CNRS UMR 7335, 06560 Valbonne, France \\ ${ }^{2}$ Departament de Fisica, Universitat de les Illes Baleares, 07122 Mallorca, Spain \\ ${ }^{3}$ Institut Mediterrani d'Estudis Avançats, CSIC-UIB, E-07071 Palma de Mallorca, Spain
}

(Received 10 February 2014; published 3 June 2014)

\begin{abstract}
We investigate the relationship between passive mode locking and the formation of time-localized structures in the output intensity of a laser. We show how the mode-locked pulses transform into lasing localized structures, allowing for individual addressing and arbitrary low repetition rates. Our analysis reveals that this occurs when (i) the cavity round-trip is much larger than the slowest medium time scale, namely the gain recovery time, and (ii) the mode-locked solution coexists with the zero intensity (off) solution. These conditions enable the coexistence of a large quantity of stable solutions, each of them being characterized by a different number of pulses per round-trip and with different arrangements. Then, each mode-locked pulse becomes localized, i.e., individually addressable.
\end{abstract}

Localized structures (LS) are confined states appearing in nonlinear dissipative systems having a large aspect-ratio. They are characterized by a correlation range much shorter than the size of the system, making them individually addressable objects. LS may form when two different solutions coexist for the same values of the parameters, although more complex scenarios exist, see [1,2] for a review. The most common case consists in the coexistence of a homogeneous and a modulated solution $[3,4]$.

Spatial LS have been widely observed in nature in systems like granular media [5], gas discharges [6], semiconductor devices [7], reaction-diffusion systems [8], fluids [9], convective systems [10] and optical cavities [11]. The possibility of using LS as information bits for processing information in optical devices [12-14] has attracted an increasing interest in the last twenty years. LS have been observed in the transverse section of broad-area semiconductor microcavities injected by a coherent electromagnetic field [15] (passive morphogenesis) and are also termed "cavity solitons." More recently, spatial LS have been observed in laser systems where they arise from spontaneous emission noise [16,17] (active morphogenesis), without requiring an injected field. Because these lasing LS appear in a phase invariant system, their dynamical ingredients and their properties are very different from the LS appearing in injected resonators [18].

Recent works have addressed the question whether the concept of LS can be extended to the time domain [19-22] in the case of optically injected cavities. Here we propose to answer to this question considering a phase invariant system, namely a passively mode-locked laser. Passive mode locking (PML) is an elegant method leading to the emission of pulses much shorter than the cavity round-trip. It is achieved by combining two elements, a laser amplifier providing gain and a nonlinear loss element, usually a saturable absorber (SA). The different dynamical properties of the SA and of the gain create a window for regeneration only around the pulse.

PML can be successfully described via the seminal Haus' master equation, which combines the nonlinear Schrödinger equation with dynamical nonlinear gain and losses [23]. In fiber or Ti:sapphire lasers [24], for which the gain and the absorption are respectively much slower and faster than all the other variables, the Haus equation can be approximated by the subcritical cubic-quintic complex Ginzburg-Landau equation where one replaces for simplicity the slowly evolving net gain-which has a typical time scale of $\Gamma^{-1}=10 \mathrm{~ms}$ in doped fibers - by a constant. This constant must be determined self-consistently as it depends on the number of PML pulses per round-trip, which may be one (fundamental PML) or $N_{h}$ ( $N$-th order harmonic PML). The stability of these different emission states is described by the so-called background stability criterion of PML [25], which states that a pulsating solution is stable only if the losses are larger than the gain during the whole interval between pulses. Accordingly, the gain constant of the stable solution must be negative, leading to a well defined value of $N_{h}$ for a given value of the pump and of the round-trip. The PML pulses are interpreted as dissipative solitons, see [26] for a review, and they are sometimes studied as perturbed nonlinear Schrödinger conservative solitons.

Noteworthy, a similar regime exists in monolithic semiconductor PML lasers in which the cavity round-trip $(\tau \sim 10 \mathrm{ps})$ is much shorter than the gain recovery time scale $\Gamma^{-1} \sim 1$ ns and where the gain weakly evolves around its equilibrium value. On the other hand, in external cavity configurations [27,28], the semiconductor fast time scales allow exploring the transition from the regime $\Gamma \tau \ll 1$ toward $\Gamma \tau \gg 1$, which would be impractical with other active materials. 
In this paper we describe both theoretically and experimentally how lasing LS form out of a PML semiconductor laser during the transition from the regime $\Gamma \tau \ll 1$ toward $\Gamma \tau \gg 1$, thus clarifying in which conditions the pulses becomes localized and can be used as independent bits for storing information. We show that LS require two conditions to be fulfilled: (i) the trivial off solution must be stable and coexist with the mode-locked solution and (ii) the cavity round-trip must be much longer than the slowest medium time scale (i.e., the semiconductor gain). While condition (ii) usually implies the violation of the background stability criterion for fundamental mode locking and leads to a high order harmonic mode-locked solution $\left(N_{h} \gg 1\right)$ as the unique stable solution, we show that, if condition i) is verified, the background automatically becomes stable. This enables the coexistence of a large quantity of stable solutions, each of them being characterized by a different number of pulses per round-trip (ranging from zero to $N_{h}$ ) with different arrangements. In this situation, the PML pulses becomes LS that can be addressed as independent bits.

Our observations generalize to lasing systems the alloptical buffer based on a Kerr fiber resonator [19,20]. Systems based on lasing LS have the advantage of avoiding the use of an injection beam and bypassing the associated tuning problems. Besides, the phase invariance of our system opens the way to exploit the phase degree of freedom for additional encoding of information. In terms of applications, semiconductor devices as support of phase invariant all-optical buffers are very attractive allowing for fast time scale and scalability. Exploiting lasing LS in an arbitrarily long cavity results in laser pulses at an arbitrarily low repetition rate, with a reconfigurable pulse pattern, which may be very useful for applications that necessitate pulses on demand like, e.g., broadband spectroscopy, telecommunications and astronomy.

We describe the PML laser using the generic delayed differential equation model of [29] which generalizes Haus's model as it encompasses both the pulsating and steady regimes. Denoting by $A$ the amplitude of the optical field, $G$ the gain, and $Q$ the saturable losses, the model reads

$$
\begin{gathered}
\frac{\dot{A}}{\gamma}=\sqrt{\kappa} \exp \left[\frac{\left.(1-i \alpha) G_{\tau}-(1-i \beta) Q_{\tau}\right] A_{\tau}-A,}{2},\right. \\
\dot{G}=g_{0}-\Gamma G-e^{-Q}\left(e^{G}-1\right)|A|^{2}, \\
\dot{Q}=q_{0}-Q-s\left(1-e^{-Q}\right)|A|^{2},
\end{gathered}
$$

where time has been normalized to the SA recovery time, $\alpha$ and $\beta$ are the linewidth enhancement factor of the gain and absorber sections, respectively, $\kappa$ the fraction of the power remaining in the cavity after each round-trip, $g_{0}$ the pumping rate, $\Gamma$ the gain recovery rate, $q_{0}$ is the value
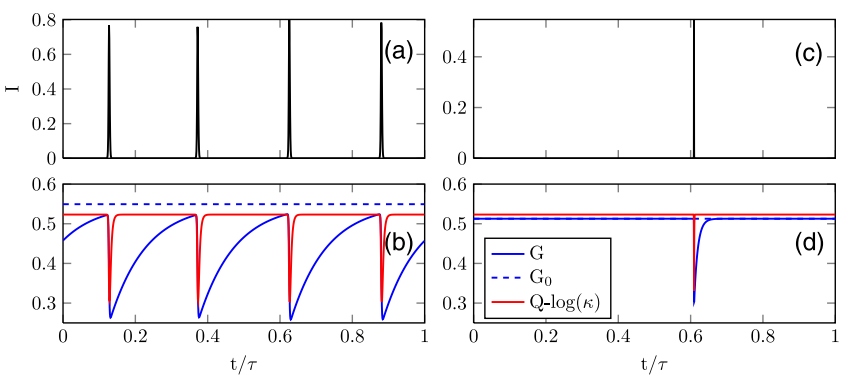

FIG. 1 (color online). Intensity of the field (a),(c), gain and total losses (b),(d). For short delay $\tau=10 \Gamma^{-1}$ and $G_{0}=1.1 G_{\text {th }}$ (a),(b) the intensity and the absorption $(I, Q)$ reach an equilibrium between pulses but not the gain that remains far from the equilibrium value $G_{0}$. For $\tau=100 \Gamma^{-1}$ and $G_{0}=0.98 G_{\text {th }}$ (c), (d) all the variables reach equilibrium and the solution becomes localized. The parameters are $\alpha=\beta=0, \kappa=0.8, \quad s=3$, $q_{0}=0.3, \Gamma^{-1}=25$ and $\gamma=10$.

of the unsaturated losses which determines the modulation depth of the SA, $s$ the ratio of the saturation energy of the SA and of the gain sections and $\gamma$ is the bandwidth of the spectral filter. In Eq. (1), the subscript $\tau$ denotes a delayed value of the variable, $x_{\tau}=x(t-\tau)$. This delay renders the dynamical system infinite-dimensional and it describes the spatial boundary condition of a cavity closing onto itself. As such, it governs the fundamental repetition rate of the PML laser.

We use standard parameters values, see caption of Fig. 1. Importantly, we stress that the dynamical scenario presented in this manuscript is mostly independent of the phase-amplitude couplings. Hence, we choose $\alpha=\beta=0$ for the sake of simplicity. The lasing threshold is determined by the pump level $g_{0}$ for which the off solution $(A, G, Q)=\left(0, \Gamma^{-1} g_{0}, q_{0}\right)$ becomes linearly unstable; in our case, it is given by $g_{\mathrm{th}}=\Gamma G_{\mathrm{th}}=\Gamma\left(q_{0}-\log \kappa\right)$. Above threshold, $g_{0}>g_{\text {th }}$, a stable continuous wave (cw) solution bifurcates from the off state, but rapidly becomes unstable as $g_{0}$ is further increased. At that point, a pulsing solution emerges from it leading to PML through a supercritical Andronov-Hopf bifurcation.

The common settings for achieving PML in semiconductor lasers usually consider a SA with a relatively large modulation depth $\left(q_{0} \sim 0.3\right)$ and a cavity round-trip shorter than the gain recovery time $\tau \ll \Gamma^{-1}$. With these two conditions, the fundamental PML solution appears above the solitary laser threshold and exhibits a single pulse per round-trip. As the pump or the cavity length is increased, the so-called regime of harmonic mode locking develops at the expense of the fundamental PML solution. Such transition can be understood via the background stability criterion. Harmonic mode locking appears when this condition is not verified anymore for the fundamental regime. The increases of the pumping current or of the cavity length lead to an increasing number of pulses $N_{h}$ in a single round-trip, such that the newfound value of $N_{h}$ 
becomes again compatible with the background stability criterion. This mechanism is illustrated in Figs. 1(a) and 1(b), where $N_{h}=4$. One notices in Fig. 1(b) that the gain (blue line) that tends to $G_{0}$ (dotted line) never surpasses the losses (red line). It is useful to stress that, for a given value of pumping current and $\tau$, only one of these harmonic PML solutions is usually stable [25,29] notwithstanding a small region of bistability in the transition region between operation at harmonic number $N_{h}$ and $N_{h}+1$.

The scenario described above radically changes for a long cavity, still choosing a SA with a strong modulation depth. We analyzed in Fig. 2 the PML bifurcation scenario with DDE-BIFTOOL [30]. For a relatively short cavity, see Fig. 2(a), fundamental PML still occurs as a supercritical Andronov-Hopf bifurcation on the dominant $\mathrm{cw}$ lasing mode and PML exists only above its bifurcation point up to a pump level where it becomes unstable against the harmonic PML solution $N_{h}=2$ (not shown). Yet, for longer cavities, see Fig. 2(b), the Andronov-Hopf bifurcation that originates the PML state becomes subcritical; hence, the PML solution may exist below its bifurcation point, where it coexists with the $\mathrm{cw}$ solution. For even
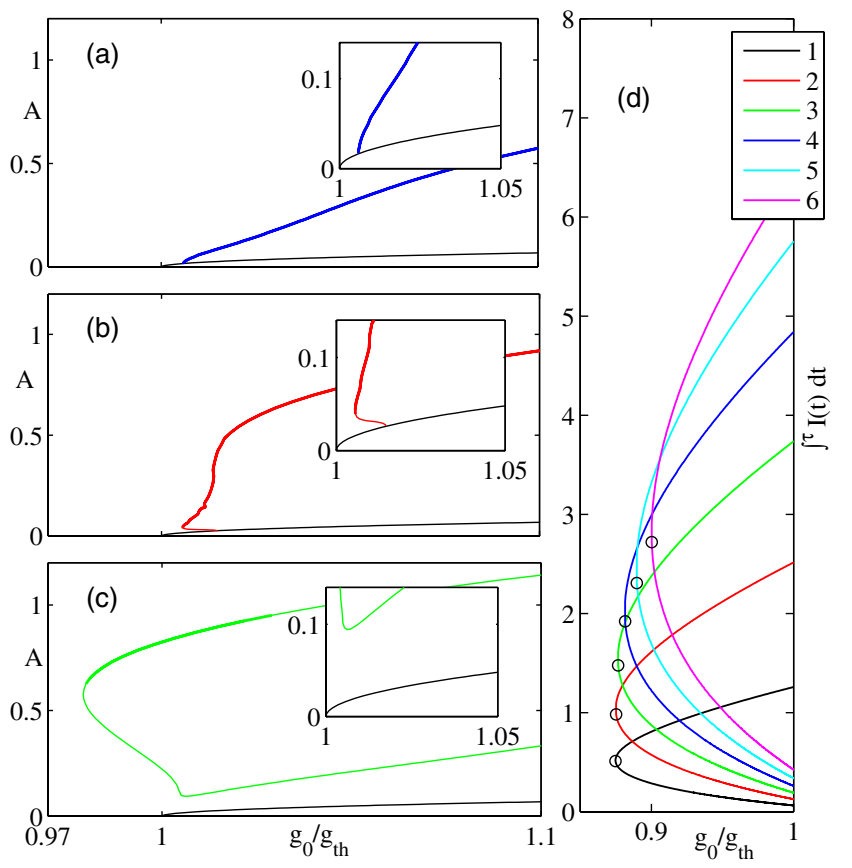

FIG. 2 (color online). Panels (a)-(c) depict the bifurcation scenario as a function of the gain for different values of the delay: (a) $\tau=1.2 \Gamma^{-1}$, (b) $\tau=2 \Gamma^{-1}$, and (c) $\tau=4 \Gamma^{-1}$. For short delays, the PML periodic solution (in color) appears as a supercritical Andronov-Hopf bifurcation of the dominant $\mathrm{cw}$ mode (thin black line). The stability of the PML solution is indicated by thick lines while the stability of the cw mode is omitted. Panel (d) shows, for $\tau=16 \Gamma^{-1}$, the folding of several PML solutions having a different number of equally separated pulses per round-trip while the folding point $g_{\mathrm{sn}}$ is represented by a circle. The other parameters are like in Fig. 1. longer cavities, the breadth of the folded fundamental PML solution may very well extend below the lasing threshold, where it coexists with the off solution, see Fig. 2(c). Interestingly, during this folding phenomenon the fundamental PML branch eventually disconnects from the $\mathrm{cw}$ solution. It entails that PML appears for long delays as a saddle-node bifurcation of limit cycles instead of a nascent Andronov-Hopf bifurcation of the cw solution making this scenario out of the reach of any weakly nonlinear analysis.

The change in dynamical scenario that occurs in Fig. 2(c) has a profound consequence on the mode-locked solutions, as it can be seen in Figs. 1(c) and 1(d): the fundamental PML solution becomes stable even in the limit $\Gamma \tau \gg 1$ and the pulse, whose duration becomes dominated by the gain recovery time $\left(\tau_{p} \sim 3 \Gamma^{-1}\right)$, becomes temporally localized in the same limit. Moreover, a very large number of pulsing solutions with different number of pulses per round-trip and different arrangement become stable for the same parameter values.

We reconstructed analytically some of these solutions using New's approximation [25] for $\tau=16 \Gamma^{-1}$ and restricting our analysis to equally spaced pulses solutions, see Fig. 2(d). Clearly, all these branches of solutions extend well below the laser threshold, where they stably coexist among them and with the off solution, although New's approximation of neglecting spectral filtering leads to an overestimation of the breadth of the PML region below threshold. We calculated the expression of the folding point $g_{\text {sn }}$ shown in Fig. 2(d). Such expression, in the long delay limit $\tau \gg 1$ and for large saturation $s \gg 1$, reads

$$
\frac{g_{\mathrm{sn}}}{g_{\mathrm{th}}}=\frac{\kappa-1}{\kappa\left(q_{0}-\log \kappa\right)} W_{-1}\left[-\exp \left(\frac{q_{0}}{s} \frac{\kappa}{\kappa-1}-1\right)\right]
$$

with $W_{k}(z)$ the Lambert function. When $g_{\text {sn }} / g_{\text {th }}<1$, PML becomes stable below the unsaturated laser threshold, and the region of coexistence between the off and the PML solutions increases when $q_{0}$ and $s$ increase and/or in the good cavity limit $\kappa \rightarrow 1$, for which we simply have $g_{\mathrm{sn}}=g_{\mathrm{th}} / s$. Here, for the parameters in Fig. 2(d) one finds $g_{\mathrm{sn}}=0.89 g_{\mathrm{th}}$, in good agreement with the value calculated numerically and shown in Fig. 2(d).

The multistability evidenced by Fig. 2(d) suggests, in analogy to spatial LS [4,31], that the harmonic modelocked solution of maximal order that exists below threshold becomes fully decomposable, since essentially any pulse of this solution can be set on or off. We give evidence of this property by imposing an arbitrary sequence of pulses within the laser cavity and examining the stability of the resulting configuration. The addressing is realized by setting the system on the off solution and sending short light pulses inside the resonator. Following the tradition of [19], the bit sequence corresponds, in our case, to the letters UIB-INLN encoded over bytes of five bits. Each pulse optically injected triggers the emission of a lasing LS, 

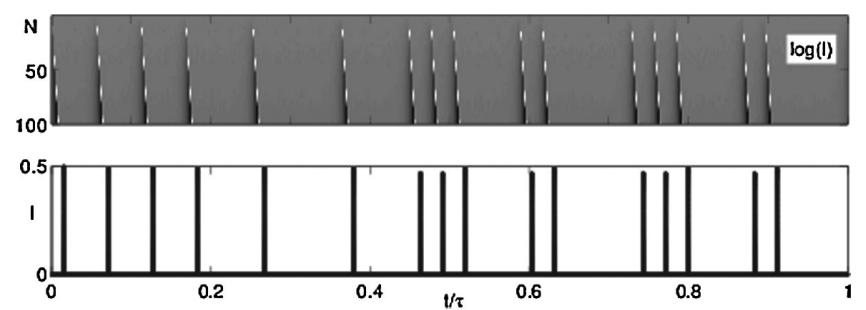

FIG. 3. Evolution over $N=100$ round-trips of a bit pattern written optically by injecting 1 ps pulses in the cavity (top) and detail over a single period (bottom). Parameters as in Fig. 1 with $\tau=200 \Gamma^{-1}$. The bit sequence is 10101010010001001110011000111001100 .

whose shape stabilizes after a short transient. A single localized pulse is not affected by a neighbor one, provided that their time separation is sufficiently large, i.e., $\Delta t \gtrsim \tau_{p}$. As can be seen in Fig. 3, the sequence remains stable, and the device acts as an all-optical buffer with a bit rate limited by the size of the LS, i.e. approximately $1 \mathrm{~Gb} / \mathrm{s}$ for typical semiconductor parameters. Erasing and writing the bits can in principle be done incoherently via cross-gain modulation in the gain and the SA sections, respectively, but this necessitates a more complex dynamical model like, e.g., [32] and will be the topic of further studies.

The results of the above theoretical analysis are confirmed by an experiment based on a Vertical Cavity Surface-Emitting laser (VCSEL) coupled to a distant semiconductor based Resonant Saturable Absorber Mirror (RSAM), as shown in Fig. 4. The VCSEL is $200 \mu \mathrm{m}$ diameter broad-area device lasing at $980 \mathrm{~nm}$ and manufactured by ULM photonics [33]. Its stand-alone threshold $\left(J_{\mathrm{st}}\right)$ is about $380 \mathrm{~mA}$. The RSAM is manufactured by BaTop and it has a peak absorption of $99 \%(1 \%$ reflection) at cavity resonance, which can be tuned by controlling the RSAM temperature. When saturated its reflection increases to $60 \%$. The full width at half maximum (FWHM) of the RSAM cavity bandwidth is $16 \mathrm{~nm}$, its saturation fluence is at $15 \mu \mathrm{J} / \mathrm{cm}^{2}$, and its relaxation time is 1 ps.

Both the VCSEL and the RSAM are mounted on temperature controlled substrates which allow for tuning the resonance frequency of each cavity; parameters are set for having the emission of the VCSEL resonant with the RSAM at the VCSEL operating current. The light emitted by the VCSEL is collected by a large numerical aperture (0.68) aspheric lens and the similar lens is placed in front of the RSAM. A 10\% reflection beam splitter allows for light extraction from the external cavity and the output is monitored by a $33 \mathrm{GHz}$ scope coupled with a $10 \mathrm{GHz}$ fast detector.

In order to match the conditions for observing LS, we set the cavity round-trip to $\tau=15.4 \mathrm{~ns}$, corresponding to a free spectral range of $65 \mathrm{MHz}$ and to $\tau \sim 50 \Gamma^{-1}$. Moreover the VCSEL is biased at $J<J_{\mathrm{st}}$. Narrow laser pulses are

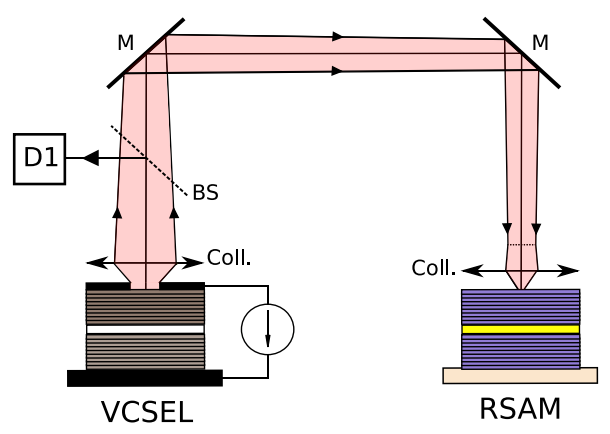

FIG. 4 (color online). Experimental setup: temperature-stabilized VCSEL and RSAM. Coll.: aspheric lens, BS: beam splitter, M: mirror, and D1: detector.

regularly emitted at the fundamental repetition rate of $65 \mathrm{MHz}$, see Fig. 5(a) which is, to the best of our knowledge, the smallest repetition rate ever obtained with a PML semiconductor laser. The pulse width is below the limit of our detection system but the corresponding spectral emission consists of a peak $0.12 \mathrm{~nm}$, (FWHM) broad, thus indicating a pulse width of approximately $12 \mathrm{ps}$. Several emission states coexist for the same values of bias current, as shown in Fig. 5. The system may remain in the off state that corresponds to the lower value between pulses or emit a pulse train with different number of pulses (from one up to nineteen) per round-trip. In these emission states, the individual pulses may appear grouped [Fig. 5(b)] or equally separated [Fig. 5(c)]. All these regimes coexist for a wide range of the VCSEL current $J$. The multistability of our system is depicted in Fig. 5(e) where we classify the different solutions in terms of the number of pulses per round-trip. It is important to remember that, for a given number of pulses, an arbitrary number of different arrangement of these pulses within the round-trip was found, corresponding to an arbitrary number of different solutions. Fig. 5(e) is obtained increasing the parameter $J$ from $J=210 \mathrm{~mA}$, where only the steady off solution is stable,
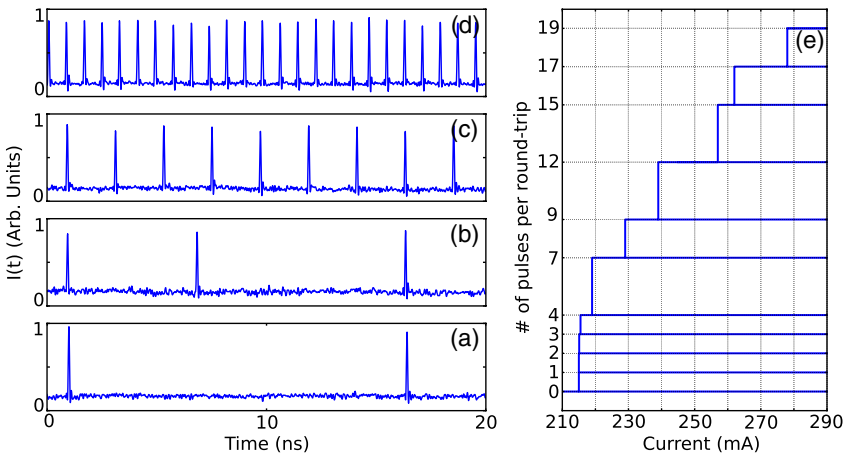

FIG. 5 (color online). Panels (a), (b), (c), and (d): coexisting time output traces $(J=290 \mathrm{~mA})$. Panel (e): experimentally obtained bifurcation diagram for the number of pulses per round-trip. The stability of each solution is indicated by the solid horizontal lines. 
up to the value where it loses its stability $(J=600 \mathrm{~mA})$ and then sweeping it down until a periodic emission with 19 pulses per round-trip appears, which probably correspond to the highest order harmonic mode-locking solution that can be fitted in our cavity round-trip [Fig. 5(d)]. As $J$ is decreased this solution looses its stability and the number of pulses per round-trip reduces progressively, until only one remains [Fig. 5(a)], thus showing the same bifurcation scenario predicted in Fig. 2(d). Each solution is spontaneously appearing as $J$ is scanned downward and, once a new solution is found, we increase $J$ to explore the stability of this solution up to $J=290 \mathrm{~mA}$. As long as the system remains on the same branch there are no changes in the arrangement of the pulses; even if several arrangements are possible, a given arrangement of $N$ pulses/round-trip is stable versus parameter variation.

In conclusion, we have shown theoretically and experimentally that PML lasers with large temporal aspect-ratio, i.e., having a cavity round-trip much larger than the other system time scales, can display multistability among the off solution and a large ensemble of different pulsing solutions. In such conditions, each pulse can be independently addressed, which allows us to interpret these pulses as phase invariant (lasing) localized structures.

We acknowledge useful discussions with S. Barland. J. J. acknowledges financial support from Ramón y Cajal program and CNRS for supporting a visit at the INLN where part of his work was developed. J. J. and S. B. acknowledge financial support from project RANGER (TEC2012-38864- C03-01) and from Direcció General de Recerca de les Illes Balears cofunded by the European Union FEDER funds. INLN group acknowledges funding from Région PACA with the Projet Volet Général 2011 GEDEPULSE.

*julien.javaloyes@uib.es

[1] L. A. Lugiato, Nonlinear Optical Structures, Patterns, Chaos, Chaos, Solitons, and Fractals Vol. 4 (Pergamon, New York, 1994), p. 1251.

[2] P. Mandel and M. Tlidi, J. Opt. B 6, R60 (2004).

[3] S. Fauve and O. Thual, Phys. Rev. Lett.. 64, 282 (1990).

[4] P. Coullet, C. Riera, and C. Tresser, Phys. Rev. Lett. 84, 3069 (2000).

[5] P. B. Umbanhowar, F. Melo, and H. L. Swinney, Nature (London) 382, 793 (1996).

[6] Yu. A. Astrov and H.-G. Purwins, Phys. Lett. A 283, 349 (2001).

[7] F.-J. Niedernostheide, M. Arps, R. Dohmen, H. Willebrand, and H.-G. Purwins, Phys. Status Solidi B 172, 249 (1992).

[8] K.-J. Lee, W. D. McCormick, J. Pearson, and H. L. Swinney, Nature (London) 369, 215 (1994).
[9] Junru Wu, Robert Keolian, and Isadore Rudnick, Phys. Rev. Lett. 52, 1421 (1984).

[10] E. Moses, J. Fineberg, and V. Steinberg, Phys. Rev. A 35, 2757 (1987).

[11] L. A. Lugiato, IEEE J. Quantum Electron. 39, 193 (2003).

[12] N. N. Rosanov and G. V. Khodova, Opt. Spectrosc. 65, 449 (1988).

[13] M. Tlidi, P. Mandel, and R. Lefever, Phys. Rev. Lett. 73, 640 (1994).

[14] W. J. Firth and A. J. Scroggie, Phys. Rev. Lett. 76, 1623 (1996).

[15] S. Barland, J. R. Tredicce, M. Brambilla, L. A. Lugiato, S. Balle, M. Giudici, T. Maggipinto, L. Spinelli, G. Tissoni, T. Knodl, M. Miller, and R. Jager, Nature (London) 419, 699 (2002).

[16] Y. Tanguy, T. Ackemann, W. J. Firth, and R. Jäger, Phys. Rev. Lett. 100, 013907 (2008).

[17] P. Genevet, S. Barland, M. Giudici, and J. R. Tredicce, Phys. Rev. Lett.. 101, 123905 (2008).

[18] P. Genevet, S. Barland, M. Giudici, and J. R. Tredicce, Phys. Rev. Lett.. 104, 223902 (2010).

[19] F. Leo, S. Coen, P. Kockaert, S. P. Gorza, P. Emplit, and M. Haelterman, Nat. Photonics 4, 471 (2010).

[20] T. Herr, V. Brasch, J. D. Jost, C. Y. Wang, N. M. Kondratiev, M. L. Gorodetsky, and T. J. Kippenberg, Nat. Photonics 8, 145 (2014).

[21] M. Tlidi and L. Gelens, Opt. Lett.. 35, 306 (2010).

[22] M. Tlidi, L. Bahloul, L. Cherbi, A. Hariz, and S. Coulibaly, Phys. Rev. A 88, 035802 (2013).

[23] H. A. Haus, IEEE J. Sel. Top. Quantum Electron. 6, 1173 (2000).

[24] M. J. Lederer, B. Luther-Davies, H. H. Tan, C. Jagadish, N. N. Akhmediev, and J. M. Soto-Crespo, J. Opt. Soc. Am. B 16, 895 (1999).

[25] G. New, IEEE J. Quantum Electron. 10, 115 (1974).

[26] P. Grelu and N. Akhmediev, Nat. Photonics 6, 84 (2012).

[27] R. Paschotta, R. Häring, A. Garnache, S. Hoogland, A. C. Tropper, and U. Keller, Appl. Phys. B 75, 445 (2002).

[28] D. Lorenser, H. J. Unold, D. J. H. C. Maas, A. Aschwanden, R. Grange, R. Paschotta, D. Ebling, E. Gini, and U. Keller, Appl. Phys. B 79, 927 (2004).

[29] A. G. Vladimirov and D. Turaev, Phys. Rev. A 72, 033808 (2005).

[30] K. Engelborghs, T. Luzyanina, and G. Samaey, "DDEBIFTOOL v. 2.00: A MATLAB Package for Bifurcation Analysis of Delay Differential Equations," KU Leuven, Leuven, Belgium, 2001.

[31] P. Coullet, C. Riera, and C. Tresser, Chaos 14, 193 (2004).

[32] J. Javaloyes and S. Balle, "FreETwM: A Simulation Tool for Multisection Semiconductor Lasers," http://onl.uib.es/ softwares, 2012; Phys. Rev. A 81, 062505 (2010); IEEE J. Quantum Electron. 46, 1023 (2010).

[33] M. Grabherr, R. Jager, M. Miller, C. Thalmaier, J. Herlein, R. Michalzik, and K. J. Ebeling, IEEE Photonics Technol. Lett. 10, 1061 (1998). 